\title{
EL LABORATORIO: UN ESPACIO DE FORMACIÓN EN LA ENSEÑANZA DE LAS CIENCIAS NATURALES
}

ARMÚA, A. C. ${ }^{(*)}$; CUBILLA, M.V..$^{(*) 2}$; VARGAS, S..$^{(* 3}$ y BLANCO, C. ${ }^{(* 4}$

Palabras claves: Trabajo Práctico - Laboratorio - Espacio de Formación- Articulación nivel medio y superior.

Resumen: Con el desarrollo de este trabajo se dan a conocer y se expresan los resultados obtenidos desde la Asignatura de Didáctica de la Biología y Práctica de Residencia como parte de su participación en el Proyecto de Extensión, la Universidad en el Medio, el cual tuvo inicio en el año 2016 y continúo con la segunda parte en el año 2017. El mismo consistió en el uso y restructuración de los Trabajos Prácticos de laboratorio y buscar otros espacios de formación en las Ciencias Naturales a través de experiencias sencillas, más específicamente en la asignatura Biología, en instituciones de nivel medio de gestión pública y privada de la ciudad de Corrientes y alrededores.

Introducción: Actualmente todas las actividades experimentales, están siendo revisadas desde una mirada crítica, Meinardi, (2010). Al enseñar ciencia, se debe orientar el trabajo de laboratorio hacia la formación de ciudadanos capaces de comprender sobre las ciencias y la actividad que desarrolla el científico, de esta manera los estudiantes podrán comprender como saben lo que saben los científicos y no aprender un distorsionado "método científico" (Duschl y Grandy, 2007). Al proponer los trabajos prácticos en las escuelas, se deben tener en cuenta las recomendaciones que señala, Furió et al (2005), presentar situaciones problemáticas abiertas, favorecer la reflexión de los estudiantes sobre el interés de las situaciones planteadas, favorecer el planteo de hipótesis y elaboración de diseños experimentales. A partir del año 2016 en el marco del proyecto que venimos desarrollando en el Programa la Universidad en el medio desde la Asignatura Didáctica de la Biología y Práctica de Residencia se trata que los docentes del nivel medio junto a los residentes incorporen a sus actividades educativas las prácticas de laboratorio, como un espacio de formación para aprender hacer ciencias. Los objetivos principales de la propuesta fueron: a) Identificar las concepciones que poseen los docentes sobre las

(*) Asignatura: Didáctica de la Biología y Práctica de Residencia. FaCENA-UNNE1

1 Dra. en Biología. acarmua@hotmail.com. Av. España 639 - (03794) 426485. Corrientes (Capital) - C.P. 3.400

2 Prof. en Biología. mariavictoriacubilla@gmail.com

3 Prof. en Biología. Susana.var14@gmail.com

4 Prof. en Biología. carolinablanco658@gmail.com 


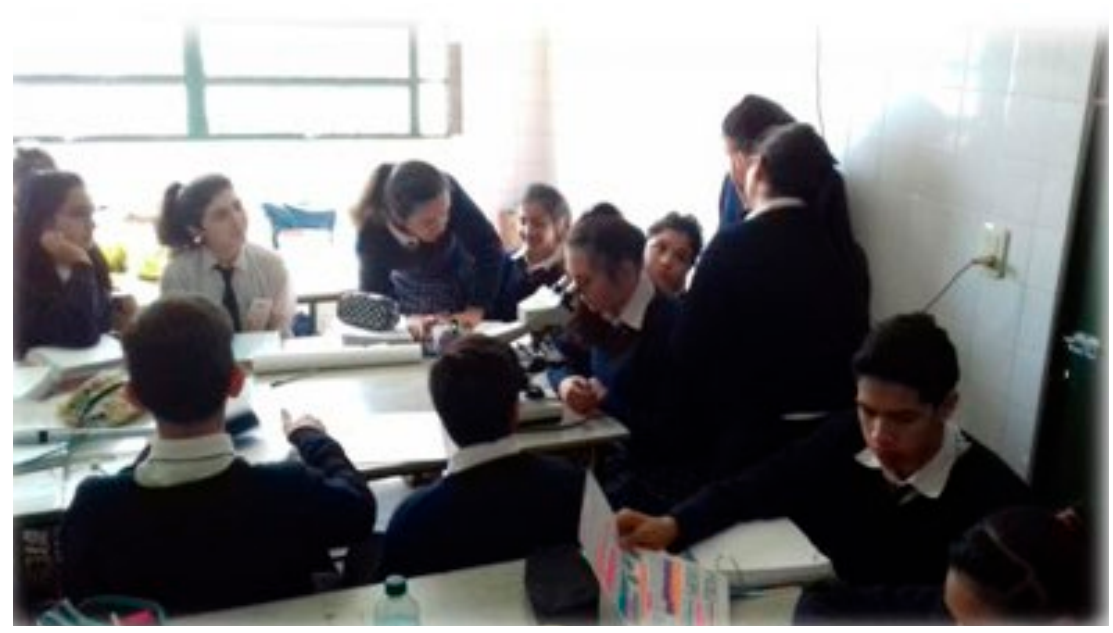

Figura1: alumnos participando en el T.P. de laboratorio Transporte a traves de la membrana plasmática

prácticas de laboratorio a través de los registros de observación, b) Incentivar en los docentes de nivel medio la implementación de trabajos prácticos de laboratorio como un espacio para que los educandos aprendan hacer y se interesen por las ciencias naturales.

El propósito de concretar este proyecto surge, por esta necesidad de replantearnos entorno a la búsqueda de espacios alternativos de enseñanza y aprendizaje que contribuyan a la comprensión de los contenidos y a la expresión de capacidades, en este caso el uso del laboratorio o de otros espacios donde manipulen instrumentos y materiales básicos, especialmente en propuestas áulicas en la asignatura biología, animando de este modo a docentes y alumnos a reintroducir, desarrollar y llevar a cabo trabajos prácticos como una estrategia favorable, enriquecedora y significativa para aprender hacer ciencia.

Metodología: Continuando con una actividad que tuvo inicio en el año 2016 y de la cual se obtuvieron resultados valiosos para mejorar, fortalecer y ampliar el campo y las propuestas de trabajo. En esta oportunidad se hicieron efectivas las propuestas en las cuatro instituciones de nivel medio que formaban parte del proyecto y otras cinco instituciones, de gestión pública y privada. En general las propuestas en cada institución se caracterizaban por:

- Abordar temáticas variadas, de las planificaciones.

- Trabajar con distintos cursos y divisiones en una misma institución.

- Utilizar estrategias y recursos diversos.

- Generar espacios para pensar, formular preguntas, que estimulen a los alumnos a trabajar en equipo, reflexionar, integrar y de esta manera ir mejorando sus producciones finales.

La ejecución de los trabajos de laboratorios se realizó como parte de las actividades propuestas durante las prácticas de residencia de los alumnos que en el ciclo lectivo 2017 se hallaban cursando la asignatura, $(f-$ gura 1). Para ello, los residentes incluyeron trabajos de laboratorio en sus planificacio- 

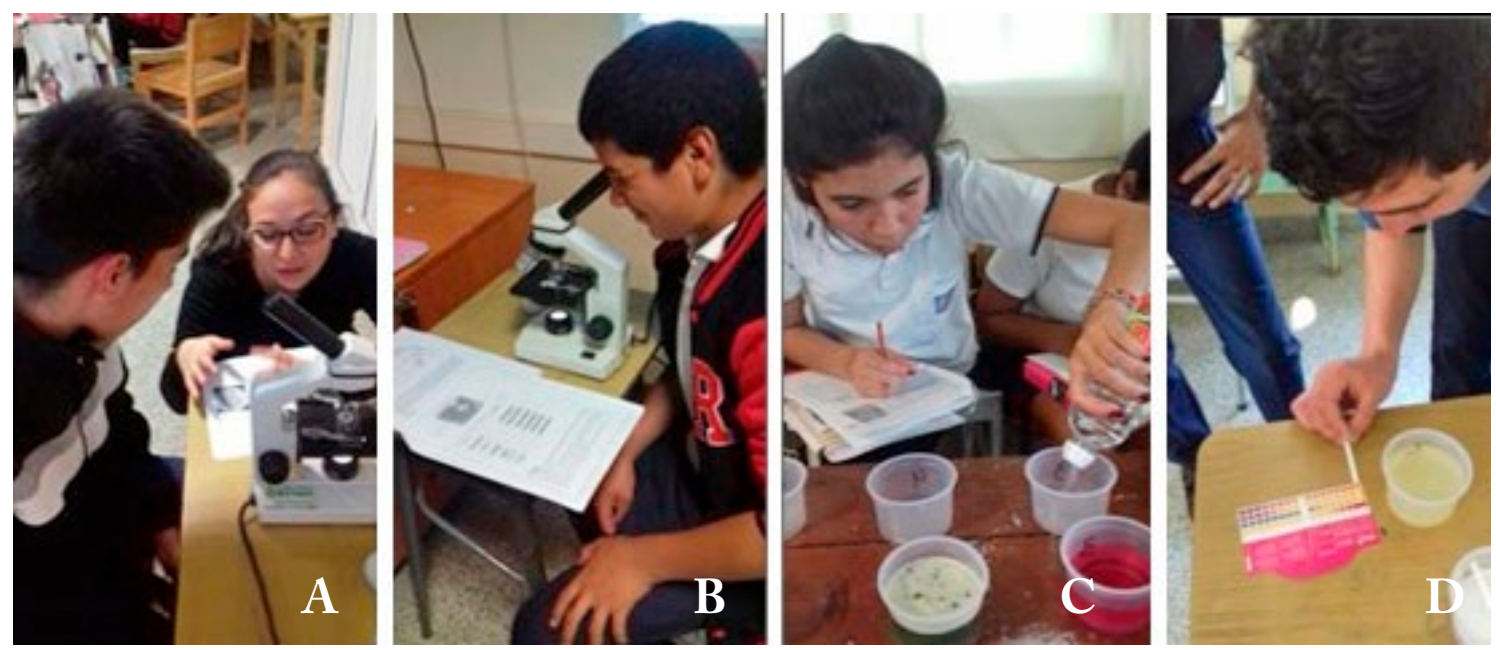

Figura 2: alumnos de 3er año participan del T. P. de laboratorio Sistema Reproductor Gónadas y células sexuales (A y B) y T. P. de laboratorio Sustancias Ácidas y Básicas (C y D)

nes de Residencia. En otras oportunidades para la elaboración del Trabajo Practico, el docente del curso proporcionaba el tema a desarrollar desde su programa.

Las instancias de trabajo para el cumplimiento de los objetivos planteados fueron los siguientes:

a. Observación y registro de clases en las instituciones de nivel medio, siguiendo una guía de las diferentes dimensiones a tener en cuenta en el desarrollo de una clase proporcionada por los docentes de la asignatura.

b. Acompañamiento de los docentes de la asignatura, diseño y elaboración de trabajos prácticos de laboratorio por parte de los residentes. (figuras 2 y 3 ).

c. Producción de materiales didácticos (guías de lectura, maquetas, modelos científicos, materiales audiovisuales, entre otros). $\mathrm{Su}-$ ministro y utilización de instrumentos básicos de laboratorio y preparados histológicos.

d. Evaluación en proceso, reconociendo las debilidades y fortalezas de los alum- nos e ir trabajando sobre estos y efectuar los ajustes y cambios necesarios en el proceso de enseñanza y aprendizaje.

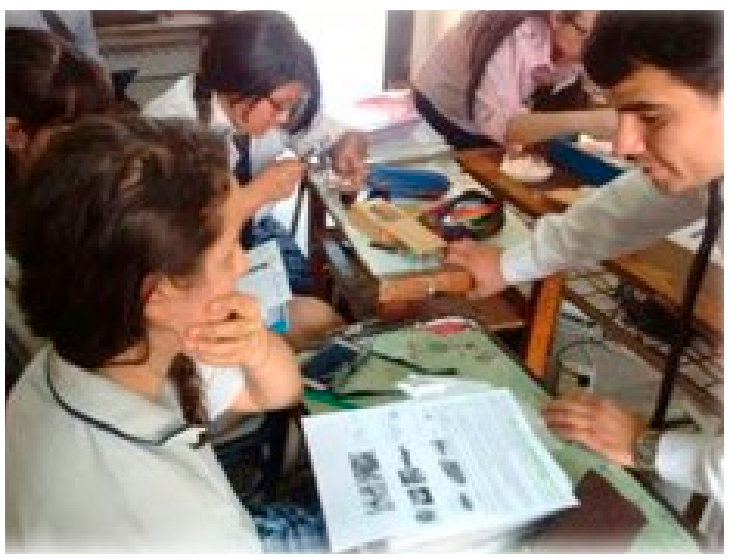

Figura 3: alumnos de 6to año participando en el trabajo Mal de Chagas: el vector

Las propuestas de este proyecto también se hicieron extensiva a otras instituciones de capital y lugares cercanos como Paso de la Patria, que en este periodo 2017 no recibieron residentes, a los efectos de ir ampliando los trabajos de laboratorio en la enseñanza de las Ciencias Naturales. En estas últimas situaciones las propuestas de traba- 
jo se elaboraron a partir de la planificación áulica del docente del curso, teniendo en cuenta principalmente lo siguiente: el tema, las actividades y los recursos institucionales disponibles.

Los trabajos prácticos se desarrollaron en el aula y el laboratorio, en aquellas instituciones que contaban con la misma. En el primer caso, se preparó un espacio determinado para la presentación del material, recursos a utilizar, se organizó el desarrollo de la clase y actividades en torno a estos espacios de trabajo (figura 3). En el segundo caso se pusieron en marcha la distribución y organización de las actividades, el tipo de trabajo que se iba a desarrollar y los recursos disponibles (figura 4). Asimismo, se presentaron casos particulares, a pedido de la docente del curso, el TP de laboratorio "Reproducción Asexual en plantas y animales", se replicó en las diferentes divisiones del 3er año, ya que consideraba que desarrollar el tema con material biológico, preparados histológicos afines, permitiendo una observación directa del proceso reproductivo, favorecía la comprensión y comparaciones de los diferentes tipos de reproducción de los seres vivos. Situación similar se dio en el TP “Células sexuales” que se concretó en dos 4to año (Figura 4 y 5 ).

Resultados: Los Residentes de la asignatura Didáctica de la Biología y Práctica de Residencia asumieron un rol protagónico en la ejecución del proyecto, lograron materializar y ser partícipes activos en la elaboración de las actividades y ulterior implementación. Asimismo, la incorporación

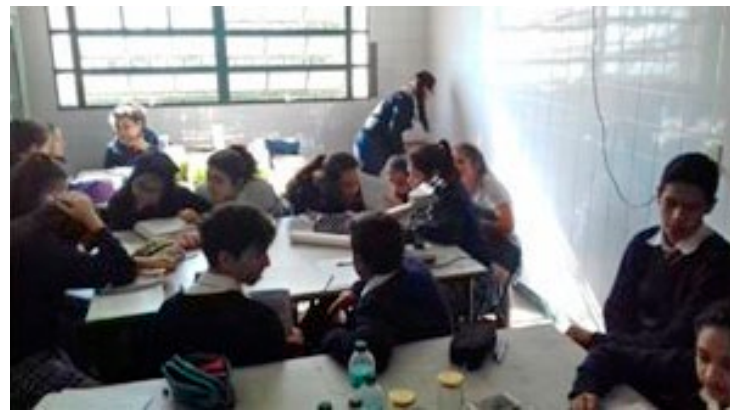

Figura 4: alumnos de 4to año participando del T. P. de laboratorio Sistema

Reproductor: Gónadas y células sexuales

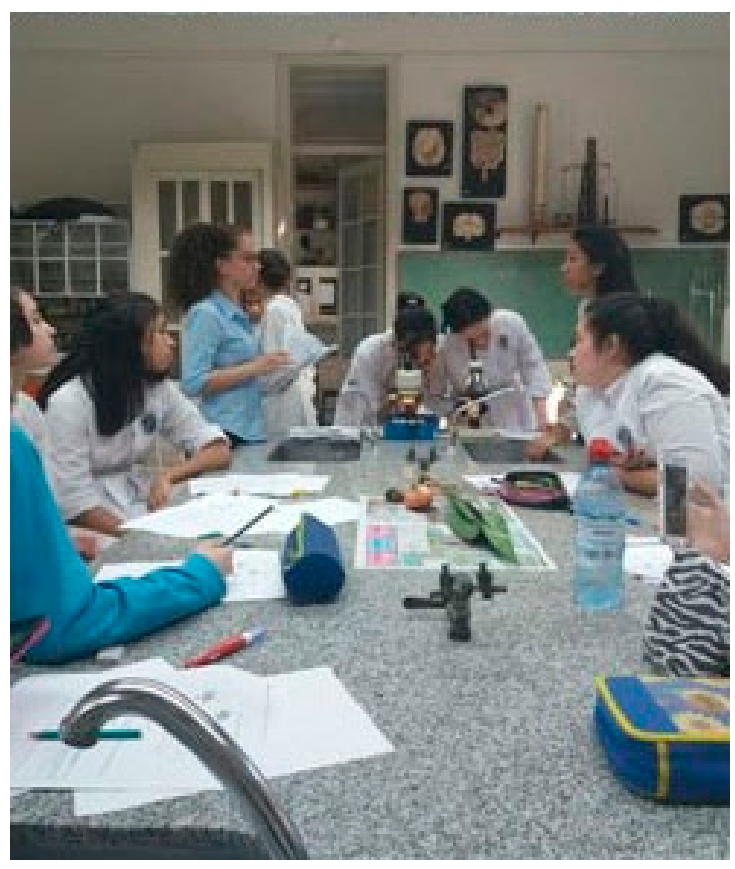

Figura 5: alumnos de 4to año participando del T. P. de laboratorio Reproducción Asexual

de los TP en el desarrollo de la mayor parte de los ejes estructurantes de la asignatura, fomentó y entusiasmó a los docentes de nivel medio a incorporarlo a su práctica sin limitaciones, ni condiciones, por el alcance y los resultados obtenidos. Reflejándose estos cambios en las producciones, evaluaciones y otras actividades de integración que los estudiantes de nivel medio efectúan. 


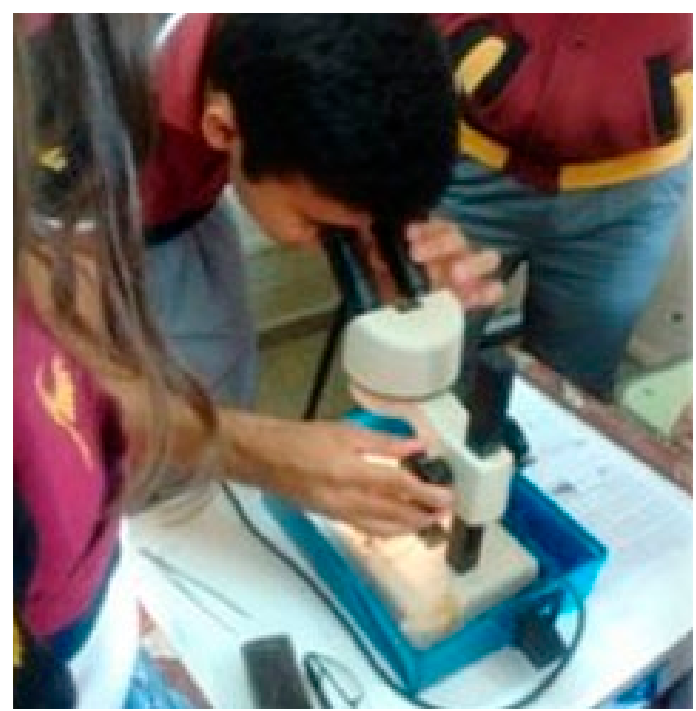

Figura 6: alumnos de 6to año reconociendo a través de la lupa las características morfológicas del vector que transporta el parásito del Mal de Chagas

Se concretaron un total de 16 propuestas de TP de Laboratorio.

A partir de la ejecución del proyecto y la experiencia adquirida a través del mismo, nos lleva a afirmar que la posibilidad de lograr espacios de trabajo dinámicos y flexibles distintos, en este caso asistir al laboratorio o transformando el aula para concretar un trabajo práctico de laboratorio, despierta en los alumnos la curiosidad, el entusiasmo por observar, descubrir, el interés, presta especial atención a lo nuevo, a lo distinto y además potencia otras habilidades que quizás antes no la ponían en juego en el proceso de aprendizaje (Figura 6 y 7).

Conclusión: Enfocar la enseñanza de las ciencias naturales con esta mirada, estimula el trabajo individual y en equipo. La capacidad del docente en la re- organización del trabajo, orientado hacia un desafío del planteo de objetivos y los medios para que los alumnos construyan su propio aprendiza-

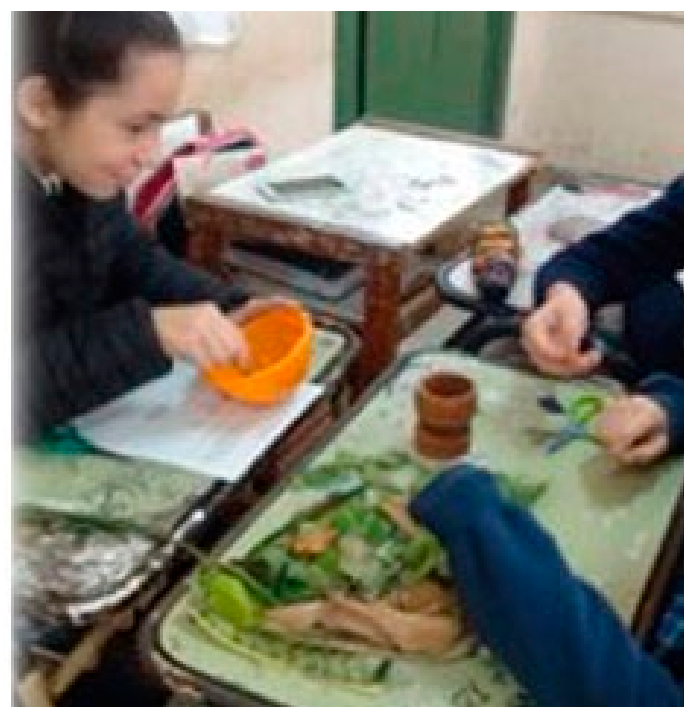

Figura 7: alumnos de 2do año participando en el T. $P$. de laboratorio Separación y extracción de Pigmentos vegetales

je como ciudadanos en el nivel medio.

Finalmente, los propósitos que nos planteamos al iniciar este proyecto, nos permite afirmar que, las propuestas de trabajo práctico de laboratorio ya sea en este lugar o en el aula deben significar un puente o un andamiaje entre la temática que se desea abordar y el modo en que mis estudiantes logran comprender utilizando lo aprendido en nuevas situaciones planteadas en las Ciencias Naturales.

\section{BIBLIOGRAFÍA}

DUSCHL, R. y R. GRANDY, 2007. Teaching scientific inquirí (The book summary). Rotterdam, The Netherlands: Sence $\mathrm{Pu}-$ blishers.

FURIO, C, J. PAYA y P. VALDES, 2005. ¿Cuál es el papel del trabajo experimental en la Educación Científica? En Gil Perez, D. (Ed) ¿Cómo promover el interés por la Cultura Científica? Santiago de Chile UNESCO.

MEINARDI, E., 2010. Educar en Ciencia. Buenos Aires. Paidos. 